\title{
Doce tesis sobre WikiLeaks
}

\author{
Geert LovinK y Patrice Riemens*
}

Traducción de Eva Aladro Vico

Propuesto: 12 de diciembre de 2010

Evaluado: 18 de diciembre de 2010

Aceptado: 20 de diciembre de 2010

(Abstracts y palabras clave al final del texto)

\section{TESIS 0}

¿Qué pienso de WikiLeaks? “¡Creo que sería una buena idea!” (basado en la famosa respuesta de Gandhi a la pregunta: ¿Qué opina usted de la civilización occidental?)

\section{TESIS 1}

\section{Cambio de medios-Los medios en proceso de cambio}

Los desarrollos tecnológicos en los medios están provocando una reestructuración fundamental del sector editorial y de los periódicos, que sitúa a los medios tradicionales en una posición de férrea competencia con los nuevos medios online, por la conquista de la superioridad en el mercado. Sin embargo el cambio mediático es algo más que una "crisis del periodismo" o la llegada del IPad: la ley de la propiedad, la libertad de expresión, y el funcionamiento de la esfera pública se ven, todos ellos, afectados por el cambio.

Las filtraciones y revelaciones son un rasgo común a muchas épocas, pero nunca antes un grupo no empresarial ni estatal había logrado algo de la importancia de lo que ha conseguido WikiLeaks, primero con el video del "asesinato colateral", después con los papeles de la Guerra de Afganistán, y ahora con el "Cablegate". Parece que hemos llegado al instante en que el proceso cuantitativo empieza a conformar un cambio cualitativo. Cuando WikiLeaks comenzó a llegar

\footnotetext{
* Geert Lovink y Patrice Riemens escriben estas tesis en agosto de 2010. Geert Lovink estudió Ciencias Políticas en la Universidad de Ámsterdam, Patrice Riemens es Geógrafo, experto en el conocimiento abierto y Software libre.
} 
a la opinión pública en 2010, ese cambio no se daba todavía. En cierto sentido, las "colosales" revelaciones de WikiLeaks pueden explicarse como una consecuencia de la dramática expansión de las tecnologías de la información junto con la espectacular caída de los costes, incluidos los derivados del almacenamiento de millones de documentos. Otro factor coadyuvante es el hecho de que la seguridad del secreto oficial y empresarial — por privados que fueran - se hace cada vez más difícil en una era de constante reproductibilidad y difusión. WikiLeaks se convierte en el símbolo de una transformación a gran escala en la "sociedad de la información", sosteniendo un espejo de cuanto va a presentarse en el futuro. Así mientras uno puede considerar WikiLeaks como un proyecto (político) y criticarlo por su modus operandi, también puede verlo como una fase "piloto" de la evolución hacia una cultura mucho más generalizada de la exposición anárquica, más allá de la tradicional política de apertura y transparencia.

\section{TESIS 2}

Para bien o para mal, WikiLeaks se ha catapultado a sí misma al ámbito de la política internacional de alto nivel. Repentinamente WikiLeaks se ha convertido en un jugador de pleno derecho, tanto en la escena mundial como en las esferas nacionales de algunos países. A pesar de su pequeñez, WikiLeaks, por la gracia de sus revelaciones, se codea con gobiernos o grandes empresas (su siguiente objetivo), - al menos en el dominio de la recolección y concentración de información, y de su difusión-. Al mismo tiempo, no está claro si se trata de un fenómeno permanente o temporal, o de algo inducido por cierta moda - WikiLeaks parece apoyar lo primero, y cada vez más esa parece ser la tónica. A pesar de ser un enclenque actor no estatal ni empresarial, su lucha contra el gobierno estadounidense está siendo canalizada adecuadamente, midiendo su capacidad de golpe de acuerdo con su peso. Podríamos llamar a este estado de la posmodernidad la "talibanización" de la teoría del "mundo plano", en el que las escalas, los tiempos y los espacios, son declarados completamente irrelevantes. Lo que cuenta es el momento de celebridad y la intensa acumulación de la atención de los medios. WikiLeaks consigue capturar la atención a través de espectaculares filtraciones de información, mientras otros actores, especialmente los grupos de la sociedad civil y las organizaciones de derechos humanos, luchan desesperadamente por conseguir difundir su mensaje. Mientras estos últimos tienden a jugar respetando las reglas y buscando la legitimidad de las instituciones dominantes, la estrategia de WikiLeaks es populista en tanto se apoya en la desafección popular hacia la política a gran escala. La legitimidad política, para WikiLeaks, ya no es un título graciosamente concedido por los poderes fácticos. WikiLeaks cortocircuita ese viejo régimen de estructuras de poder y en su lugar busca la fuente de su legitimación política en la info-sociedad de hoy: la banalidad radiante del espectáculo. WikiLeaks pone brillantemente en uso la "velocidad de escape" de las TI, utilizando las Tecnologías de la Información para dejarlas a éstas mismas atrás e irrumpir rudamente en el ámbito de las políticas del mundo real. 


\section{TESIS 3}

En la saga actual titulada "El declive del imperio americano", WikiLeaks entra en escena como el asesino de una víctima fácil. Sería difícil imaginar que alguien infligiera un daño similar a los gobiernos chino o ruso, o incluso al de Singapur, por no citar a sus socios "empresariales". En Rusia o en China, grandes barreras culturales y lingüísticas lo impiden, y no digamos las barreras del poder directo que habría que traspasar. También son importantes factores en torno a los miembros integrantes de dichas sociedades, aunque hablamos de cada vez más próximas (y claramente más globales) culturas y agendas de los hackers, info-activistas y periodistas de investigación. En este sentido, WikiLeaks en su actual manifestación sigue siendo un producto típicamente "occidental" y no puede reclamarse su validez universal o global.

\section{TESIS 4}

Una de las dificultades principales para explicar WikiLeaks surge del hecho de que no está claro (ni para la misma gente de WikiLeaks) si se ven a sí mismos y operan como proveedores de contenido, o simplemente como un conducto para datos filtrados (la impresión es que eso depende de cada contexto o circunstancias). Esto, por cierto, ha sido un problema común con la digitalización masiva de los medios medios, proceso en el que las comunicaciones y la edición se han convertido en un servicio y no en un producto. Julian Assange recula cuando se le presenta como "editor jefe" de WikiLeaks; sin embargo WikiLeaks afirma que edita el material antes de su publicación y proclama que comprueba la autenticidad de los documentos con la ayuda de cientos de analistas voluntarios. Entre los activistas de medios el debate sobre contenido o canal se viene manteniendo desde hace décadas, sin llegar a una conclusión. En lugar de intentar resolver tal inconsistencia, sería mejor buscar enfoques nuevos y desarrollar nuevos conceptos críticos de lo que se ha convertido en una práctica de difusión híbrida, que implica a actores de fuera de los dominios tradicionales de los medios informativos. Esto puede que explique por qué Assange y sus colaboradores rechazan ser etiquetados en términos de las "viejas categorías" (periodistas, "hackers" o piratas, etc.), y reivindican que suponen una nueva Gestalt en el escenario informativo mundial.

\section{TESIS 5}

Es un hecho innegable que el periodismo de investigación sufre un declive constante causado por la disminución de los fondos a él destinados. El periodismo hoy en día es poco más que un sistema de remezcla de la información proporcionada desde el exterior, elaborada por el sistema de relaciones públicas. La constante aceleración y crecimiento de la llamada economía de la atención crea además la imposibilidad de alojar las historias complicadas en el sistema comunicati- 
vo. Las empresas propietarias de los medios masivos están cada vez menos dispuestas a discutir en detalle los resultados y las políticas de la economía global neoliberal. El cambio de la información al infoentretenimiento ha sido abrazado por los mismos periodistas, dificultando la publicación de los reportajes complejos. WikiLeaks aparece en este estado de la cuestión como un outsider, alguien extraterritorial, envuelto en el halo vaporoso del "periodismo cívico", en el periodismo del "Hágalo usted mismo" que emana de la blogosfera o de medios más veloces incluso, como Twitter. Lo que plantea WikiLeaks, aunque hasta ahora ha sido incapaz de organizar, es la "subcontrata" para la interpretación de los documentos filtrados. Este trabajo, curiosamente, se deja a los pocos periodistas en nómina que quedan en unos selectos medios periodísticos "de calidad". Posteriormente los académicos recogen los restos y dan vueltas a las historias detrás de las puertas cerradas de los establos editoriales. Pero ¿qué queda del trabajo de comentario crítico por parte de una organización colectiva? Ciertamente todos estamos ocupados en nuestras críticas menores; y el caso es que WikiLeaks genera su capacidad de inspirar irritación en los grandes negocios, precisamente por su relación trasversal y simbiótica con las instituciones mediáticas establecidas. En ello hay una lección para las multitudes: sal del ghetto y conecta con el Otro edípico. Ahí radica el terreno conflictivo de lo político.

El periodismo de investigación tradicional solía fundamentarse en tres fases: el descubrimiento de los hechos, el cruce y contrastación de los datos y la contextualización de los mismos en un discurso comprensible. WikiLeaks lleva a cabo la primera fase, reclama hacer lo segundo, pero omite enteramente lo tercero. Ello es síntoma de un especial tipo de ideología del libre acceso, en el que la producción de contenido queda externalizada y en manos de entidades "de fuera" y desconocidas. La crisis del periodismo de investigación ni se entiende ni se reconoce. Queda en la oscuridad cómo se mantienen materialmente las entidades productoras: se presume simplemente que el análisis y la interpretación serán asumidos por los medios informativos tradicionales. Pero esto no ocurre automáticamente. La saga de la Guerra de Afganistán y el "Cablegate" demuestran que WikiLeaks tiene que enfrentarse y negociar con los medios tradicionales bien establecidos para asegurar suficiente credibilidad. Al mismo tiempo, estos medios difusores demuestran ser incapaces de procesar totalmente el material, filtrando inevitablemente los documentos de acuerdo con sus propias políticas editoriales.

\section{TESIS 6}

WikiLeaks es una típica SPO (Single Person Organization, Organización de Persona Única). Esto significa que la toma de iniciativas, de decisiones y la ejecución de las acciones se concentra en gran medida en las manos de una sola persona. Como los negocios de tamaño pequeño o medio, el fundador no puede ponerse en cuestión, y como en muchos colectivos, el liderazgo no rota. Esto no es infrecuente en las organizaciones, ya sea en el ámbito de la política, la cultura o en el sector de la "sociedad civil". Las SPO son muy reconocibles, emocionan- 
tes, inspiradoras, y fáciles de cubrir por los media. La sostenibilidad de una SPO depende en gran medida de las actuaciones de su líder carismático, pero su funcionamiento no se concilia bien con los valores democráticos. Por eso mismo no admiten la puesta en cuestión ni la toma de decisiones en colectivo. El "hacker" soberano Julian Assange es la cabeza identificable de WikiLeaks, y la notoriedad y la reputación de Assange se funde con la de la organización misma. Lo que WikiLeaks hace o lo que apoya, se confunde con la propia agitada vida privada - en apariencia - de Assange y también con sus poco aseadas opiniones políticas.

\section{TESIS 7}

WikiLeaks suscita la cuestión de qué tienen en común los hackers o piratas informáticos y los servicios secretos, pues existe inconfundible afinidad electiva entre ambos. La relación de amor-odio se remonta al comienzo mismo de la era de los ordenadores. No es preciso ser un fan del teórico de medios alemán Friedrich Kittler o, con ello, de las teorías conspiratorias, para reconocer que los ordenadores nacen a partir del complejo militar industrial. Desde el descifrado del código nazi Enigma por Alan Turing hasta el papel jugado por los ordenadores en la invención de la bomba atómica, pasando por el movimiento de la cibernética y por la implicación del Pentágono en el desarrollo de Internet, la articulación de la información digitalizada con la estructura militar industrial está claramente establecida. Los programadores y científicos informáticos han conformado una revolución informativa y una cultura de la apertura: pero al mismo tiempo han desarrollado la encriptación (crypto), y han cerrado el acceso a los datos para los no iniciados. Lo que algunos llaman "periodismo ciudadano" para otros es "información de guerra".

WikiLeaks es también una organización conformada intensamente por la cultura hacker de los años 80 , combinada con los valores políticos del tecno-libertarismo que emergió en 1990. El hecho de que Wiki Leaks fuera fundado - y en gran medida sigue siendo desarrollado - por geeks del núcleo duro (personas fascinadas por la tecnología y la informática) es esencial para comprender sus valores y sus movimientos. Desafortunadamente a ello acompaña una buena dosis de los aspectos menos sabrosos de la cultura hacker. No es que el idealismo, el deseo de contribuir a mejorar el mundo, esté ausente de WikiLeaks, todo lo contrario. Pero este tipo de idealismo (o si se prefiere, de anarquismo) va ligado a una preferencia por las conspiraciones, una actitud elitista y un culto (si bien condescendiente) por el secreto. Todo ello no conduce a la colaboración con personas y grupos de similar ideología, que son relegados al mero papel de simples consumidores del producto WikiLeaks. El celo misionero por ilustrar a las masas idiotas y por "revelar" las mentiras del gobierno, el ejército o las empresas, recuerda al bien conocido (e infame) paradigma cultural mediático de los años cincuenta. 


\section{TESIS 8}

La falta de simpatía con otros movimientos gemelos, de "otro mundo es posible", lleva a WikiLeaks a buscar la atención pública con revelaciones cada vez más espectaculares y arriesgadas, reuniendo con ello un grupo de partidarios generalmente pasivos pero frecuentemente de un entusiasmo salvaje. Assange mismo ha afirmado que WikiLeaks se ha desplazado voluntariamente de la blogosfera "egocéntrica" y de otros diversos medios sociales, y ahora colabora solamente con periodistas profesionales y con activistas de derechos humanos. Sin embargo, siguiendo la naturaleza y la cantidad de revelaciones de WikiLeaks desde su origen hasta el presente, nos parece que estamos ante un dispositivo de fuegos artificiales, que incluye un "gran final" en forma de máquina de apocalipsis activada, a partir de la todavía no revelada difusión de documentos "de seguridad" (".aes256"). Esto plantea serias dudas sobre la sostenibilidad a largo plazo de WikiLeaks misma, y posiblemente también del modelo de WikiLeaks. WikiLeaks opera mediante un equipo de personal ridículamente reducido - probablemente no más de una docena de personas forman el núcleo de su operación. Si la extensión y capacidad de su soporte tecnológico queda bien demostrada, la afirmación de WikiLeaks de que cuenta con algunos cientos de analistas voluntarios y expertos no se puede verificar y, para ser francos, es poco creíble. Éste es sin duda su talón de Aquiles, no solamente desde el punto de vista de su riesgo y sostenibilidad, sino políticamente también, que es lo que nos interesa aquí.

\section{TESIS 9}

WikiLeaks exhibe una asombrosa falta de transparencia en su organización interna. Su excusa de que "WikiLeaks necesita ser completamente opaca para forzar a otros a ser totalmente transparentes" es, en nuestra opinión, como aquel fabuloso cómic Espia contra Espia de la revista Mad. Se combate a la oposición de un modo que uno se vuelve indistinguible de ella. No sirve de nada proclamar la supremacía moral a posteriori, como Tony Blair hacía de modo tan excelente. Si WikiLeaks no es un grupo político ni una ONG en sentido legal, y tampoco, en este sentido, es una compañía o parte de un movimiento social, debemos investigar qué tipo de organización está tratando para nosotros. ¿Es un proyecto virtual? Después de todo, existe como un sitio web alojado con un nombre de dominio, que figura en la base de la página. ¿Pero tiene un objetivo, más allá de la ambición personal de su fundador/ores? ¿Es reproducible? ¿Vamos a ver ejemplos similares en el ámbito local o nacional? ¿Qué reglas del juego van a observar? ¿Debemos más bien considerarlo un concepto que viaja de contexto en contexto y que, como un "meme", se transforma a sí mismo en el tiempo y en el espacio?

\section{TESIS 10}

¿Quizás WikiLeaks se organizará a sí misma en torno a su propia versión del eslogan de la Fuerza Operativa de Programación de Internet, "consenso básico y 
código dominante"? Proyectos como Wikipedia o Indymedia se han resuelto cada uno de un modo en este sentido, atravesando crisis, conflictos y divisiones. Una crítica como la que hacemos aquí no intenta forzar a WikiLeaks a volverse al formato tradicional; por el contrario, intenta explorar si WikiLeaks (y sus futuros clones, asociados, avatares y miembros de familia) resistirán como modelo para nuevas formas de organización y de colaboración. El término "red organizativa" ha sido acuñado como posible nombre para dichos formatos. Otra denominación es "media tácticos". Otros aún usan el término genérico "activismo de Internet". Quizás WikiLeaks tiene otras ideas sobre la dirección que desea tomar. Pero ¿hacia dónde?. Le toca a WikiLeaks decidir por sí misma. Hasta ahora, sin embargo, hemos visto muy poco de su respuesta a esta cuestión, pues se deja a otros su planteamiento, por ejemplo en torno a la legalidad de los acuerdos financieros de WikiLeaks (Wall Street Journal).

No podemos obviar el desafío de la experimentación con las redes post-representacionales. Como el ur-blogger (bloguero primigenio) Dave Winer escribió sobre los ingenieros de Apple, "no es que tengan mala intención, es que no están bien preparados. Más que los usuarios, viven en un Campo de Distorsión de lo Real, y las personas que hacen los Ordenadores para el resto de Nosotros no tienen idea de quiénes somos ni de lo que hacemos. Pero no pasa nada, hay solución. Investiguen un poco, hagan algunas preguntas, y escuchen".

\section{TESIS 11}

La crítica ampliamente compartida del culto a la celebridad auto-inflingida de Julian Assange incita a formular alternativas. ¿No sería mejor que WikiLeaks fuera un colectivo anónimo o una "red organizativa" impersonal? Hay quien ha expresado el deseo de que haya muchas webs haciendo esta misma función. Un grupo en torno a Daniel Domscheit-Berg, que se separó de la compañía de Assange en septiembre pasado, ya trabaja en un clon de WikiLeaks. Lo que debe estudiarse detenidamente es la cantidad de conocimiento experto que exige la organización eficaz de muchos sitios web como WikiLeaks. ¿Qué caja de herramientas básicas requiere WikiLeaks? Hay, paradójicamente, un gran secreto en esta nueva manera de hacer públicas determinadas cosas. Simplemente descargar un programa de WikiLeaks y ponerlo en marcha no es una opción realista. WikiLeaks no es una aplicación de instalación como la de Wordpress para crear blogs, y la palabra "Wiki" en su denominación viene a confundir, como desde Wikipedia Jimmy Wales no hace más que señalar a voz en grito. Contrariamente a la filosofía de colaboración de Wikipedia, WikiLeaks es un negocio cerrado dirigido por un número no determinado de voluntarios sin rostro. Nos vemos forzados a reconocer que el know how necesario para desarrollar un servicio como WikiLeaks es bastante arcano. Los documentos que recibe deben ser anónimos, pero también deben convertirse en anónimos una vez que son editados on line. También se "editan" antes de difundirse a los servidores de las organizaciones informativas internacionales y a fiables e influyentes "diarios de calidad". 
WikiLeaks ha conseguido una buena cantidad de confianza y de fiabilidad con los años. Nuevas iniciativas en su campo deberán pasar por el mismo proceso lento de construcción de credibilidad. El principio de WikiLeaks no es el de piratear (al Estado o a las redes empresariales) sino el de facilitar que personas dentro de grandes organizaciones copien datos importantes y confidenciales y los pasen al dominio público, permaneciendo siempre anónimas. Si usted aspira a convertirse en un nodo de filtraciones, es mejor que se vaya familiarizando con procesos como OPSEC o seguridad de operaciones, un plan paso a paso que "identifica la información crítica para determinar si se están llevando a cabo acciones amistosas por parte de sistemas adversarios de inteligencia,o si la información obtenida por los adversarios podía interpretarse como útil para ellos y después ejecuta medidas determinadas para eliminar o reducir la explotación por parte del adversario o la información crítica amistosa" (Wikipedia). El eslogan de WikiLeaks afirma: "El coraje es contagioso". Según los expertos, la gente que pretende desarrollar una operación tipo WikiLeaks debe tener claros los riesgos que corre. La protección de las fuentes informantes es fundamental. Otro tema clave es la protección de las personas citadas en los documentos filtrados. Los documentos de Afganistán mostraron que también las filtraciones pueden causar "daños colaterales". Editar, (y borrar), es fundamental. No solamente debe haber OPSEC, sino OP-ÉTICA. Si la publicación no se hace de un modo que sea absolutamente seguro para todos los implicados, hay un riesgo claro de que esta "revolución en el periodismo" — y en la política- desencadenada por WikiLeaks se quede parada en su arranque.

\section{TESIS 12}

No creemos que tomar partido a favor o en contra de WikiLeaks sea lo más importante. WikiLeaks ha llegado para quedarse, hasta que se destruya a sí misma o sea destruida por las fuerzas que se le oponen. Nuestra idea es más bien la de intentar valorar y asegurar lo que WikiLeaks puede, podría, e incluso lo que debería hacer, ayudando a formular cómo "nosotros" podríamos relacionarnos, e interactuar con WikliLeaks. A pesar de todas sus desventajas, y contra todos los pronósticos, WikiLeaks ha prestado un brillante servicio a la causa de la democracia, la apertura y la transparencia. Como dirían los franceses, si ya existiera algo como ella, no habría tenido que ser inventada. El giro cuantitativo y - que parece que pronto será cualitativo - en la cantidad de información disponible para todos es un hecho de nuestra vida actual. No podemos esperar sino un crecimiento cada vez mayor, y más exponencial, de la información que es desvelada públicamente. Organizar e interpretar este Himalaya de datos es un desafío colectivo que aparece claramente aquí, lo llamemos "WikiLeaks" o no. 


\title{
RESUMEN
}

Los autores analizan los factores que integran el fenómeno de WikiLeaks presentando los argumentos que, a favor y en contra de dicho nuevo sistema informativo se plantean en la sociedad y para el mundo profesional del periodismo. Abogan por la calidad en el tratamiento de la información que debe ser el rasgo fundamental de los medios de comunicación, y por la vigilancia de las posibles presiones o influencias que puedan aparecer en los nuevos sistemas tecnológicos de comunicación.

Palabras clave: WikiLeaks, periodismo profesional, nuevos medios, censura, poder, sociedad, política.

\begin{abstract}
The authors analyze the features that characterize WikiLeaks phenomenon, dealing with all the pros and cons of this new information system, which affects the professional journalism as well as the society as a whole. They defend the quality of the information processing as the main feature of communication media, and for the control of the possible new pressures and influences appearing with the new communicative technologies.
\end{abstract}

Key words: WikiLeaks, professional journalism, new media, censorship, power, society, politics.

\section{RÉSUMÉ}

Les auteurs analysent les traits que caractérisent le phénomène de WikiLeaks, en présentant les arguments à faveur et en contre de ce nouvel système informatif, pour les journalistes professionels ainsi que pour la société entière. Ils se prononcent en faveur de la qualité dans le procès de l'information dans les médias, et pour la vigilance des possibles pressions et influences que peuvent apparaître avec les nouvelles technologies de la communication.

Mots clé: WikiLeaks, journalisme professionel, nouveaux médias, censure, pouvoir, société, politique. 